\section{Wissen als Ware oder öffentliches Gut?}

Wirtschaftsinteressen, Wissenschaftsinteressen und öffentliches Interesse - Eine Veranstaltung der Universitätsbibliothek zum Universitätsjubiläum 2007 der Universität Mannheim am 12. Juni 2007'

\section{Adalbert Kirchgäßner}

Die Universitätsbibliothek lud zu diesem Thema vier Referenten und zusätzlich Diskutanten zu einer abschließenden Podiumsdiskussion ein.

Den ersten Vortrag hielt Prof. Dr. Thomas Dreier vom Institut für Informationsrecht der Universität Karlsruhe zum Thema "Informationelle Mehrwertdienste - Wettbewerb durch Urheberrecht?" Professor Dreier führte aus, dass das Urheberrecht ein Persönlichkeitsrecht ist, das für die Nutzung wissenschaftlicher Erkenntnisse eigentlich kein Problem darstellt, während das daraus abgeleitete Verwertungsrecht, nämlich publizierte wissenschaftliche Erkenntnisse weiterzuverwerten, strittig ist und auch das Wettbewerbsrecht tangiert. Im digitalen Zeitalter dienen diese Rechte auch dazu, die den primären Produkten nachgelagerten Folgemärkte zu regulieren. Das Problem entsteht, wenn die Nutzung der sekundären Produkte - z.B. ein Pressedienst - dazu führt, dass die primären Produkte - z.B. Zeitungen - nicht mehr genutzt werden. Im Zeitalter der gedruckten Medien gab es eine eindeutige Wertschöpfungskette: Autor - Verleger - Händler oder Bibliothek - Leser. Hier kamen allenfalls der Buchhändler und die Bibliothek in eine Konkurrenzsituation. Da diese sich aber an verschiedene Kundenkreise wandten, war das Problem begrenzt

In den neunziger Jahren begann die "Zeitschriftenkrise", d.h. die Preise für wissenschaftliche Zeitschriften stiegen deutlich schneller als in der Vergangenheit und als die Bibliotheksetats.

Parallel dazu veränderte sich die Wertschöpfungskette durch die Digitalisierung der Medien: Die Grenzen zwischen den verschiedenen Rollen der Wertschöpfungskette verwischten und alle Teilnehmer können mit allen in Konkurrenz treten. Hinzu kommen die Mehrwertdienste, die auf den Primärprodukten aufgebaut werden können. Diese Mehrwertdienste bzw. Sekundärprodukte dienen der Befriedigung des Bedürfnisses nach Übersicht und Informationsorganisation. In der Folge muss das Urheberrecht auch die nachgeordneten Märkte berücksichtigen, die früher kaum eine Rolle spielten.

1 Die Folien der Referenten sind auf der Homepage der UB Mannheim veröffentlicht: http://www.bib.uni-mannheim.de: $8080 / \mathrm{blog} /$ p $=58$
Die Frage ist, was ist geschützt? - Das Urheberecht sieht eine Schutzfrist von 70 Jahren vor. Aber dieses verbietet nicht die Bearbeitung, nämlich die Schaffung neuer Werte aus vorhandenen Ideen. Das Ausschließlichkeitsrecht des Urheberrechts genügt hier nicht. Es muss geregelt werden, was in welchem Umfang und unter welchen Bedingungen genutzt werden darf. Es sind Schranken zu definieren, welche Handlungen zulässig sind. Diese Schranken behindern die Erstellung nachgelagerter Informationsprodukte aus Primärinformationen. Und es geht darum, was Bibliotheken ihren Nutzern anbieten dürfen. Werden diese Schranken sehr eng gefasst, behindern sie die Informationsfreiheit. In der Rechtsprechung wurde entschieden, dass Einzelinformationen aus Sammlungen systematisch und methodisch gesammelt werden dürfen, wenn sie nicht wesentliche Teile der ausgewerteten Sammlung(en) ausmachen. Folglich dürfen auch Einzelinformationen aus Datenbanken systematisch herausgesucht werden. Es besteht Übereinkunft dass die Informationsauswertung zulässig ist, aber diese Nutzung nicht dazu führen darf, dass die Verwertung der Primärquellen nicht mehr möglich ist. So dürfen z.B. Pressedienste nur als Bilder verbreitet werden, damit diese Daten nicht maschinell durchsucht werden können. Andererseits sind Links auf öffentlich zugängliche Web-Seiten zulässig.

Was nicht geregelt ist, muss durch Gerichtsentscheide geregelt werden. In Deutschland dauert es etwa sechs Jahre, bis ein letztinstanzliches Urteil gefällt ist. Dies ist zwar im internationalen Vergleich relativ schnell, aber im digitalen Zeitalter ist dies sehr lang, weil im Zweifel die angefochtene Nutzung bis dahin nicht mehr relevant ist. Die EU hat hier keine Vereinheitlichung gebracht. Sie hat 21 mögliche Schranken definiert, es aber den einzelnen Ländern überlassen, ob und in welchem Umfang die einzelnen Schranken national umgesetzt werden.

Zum Schluss schlug der Referent vor, neue Dienste zuzulassen, wenn die Nutzung der Primärquellen angemessen vergütet wird, damit die technischen Möglichkeiten genutzt werden. Aber wer soll die Vergütung regeln, wenn die Kontrahenten sich nicht einig werden?

In der Diskussion wurde benannt:

- Die Verwertung hat mit dem Urheber nichts mehr zu tun. Aber im Verlagswesen gibt es im Gegensatz zur Musik- und Filmindustrie kein eigenes Verwertungsrecht, sondern nur ein aus dem Urheberrecht abgeleitetes Verwertungsrecht.

- Ein Problem ist, dass die Hochschulen die Wissenschaftler für die Erstellung der Manuskripte bezahlen und diese in Form der Zeitschriften dann wieder kaufen sollen. Doch es gibt kaum eine Möglichkeit, dieses Verwertungsrech auf die Hochschulen zu übertragen, wie sich bei den Patenten herausgestellt hat. 
Im zweiten Beitrag sprach Herr Berndt Dugall, Leiter der Universitätsbibliothek Frankfurt, über "Die Digitalisierung wissenschaftlicher Information: Neuer Wein in alten Schläuchen oder Paradigmenwechsel?"

Im herkömmlichen Publikationsverfahren war der Reputationskreislauf zwischen Zeitschrift, Autor und Leser relativ unabhängig vom Geldkreislauf zwischen Verlagen und Bibliotheken. Im digitalen Zeitalter sind die Funktionen orts- und zeitunabhängig geworden und es entwickelten sich neue Nachfragemodelle und Vertriebsformen. Konsortial- und Nationalverträge bündeln die Nachfrage, während Lizenzen und Pay-per-View die digitale Nutzung regeln. Aber diese Veränderungen führten zu keiner Kostenersparnis. Die Preise für wissenschaftliche Zeitschriften steigen weiter mit durchschnittlich 7 Prozent und die Lizenzen sind oftmals mit Abbestellverboten belegt. Und auf der Verlagsseite erfolgte eine enorme Marktkonzentration durch Fusionen, die aber bei den Wettbewerbshütern bisher kein Interesse weckte.

Das Verhalten der Nutzer wissenschaftlicher Zeitschriften ist fachspezifisch unterschiedlich, aber über Jahre konstant: Fünf bis sieben Prozent der Titel bedienen etwa $45 \%$ der Nachfrage, die Hälfte aller Titel bedient etwa $95 \%$ der Nachfrage. Dabei ändert sich die Rangfolge der Zeitschriften im Zeitablauf nur wenig. Die Nutzung eines chemischen Artikel kostet etwa 2 Euro, während die Nutzung eines mathematischen Artikels etwa 50 Euro kostet.

Das bisherige Modell für die Einzelbeschaffung von Zeitschriften hat sich kaum verändert: Abonnement für die gedruckte Ausgabe und die Nutzung der elektronischen Ausgabe kostenfrei oder gegen Aufpreis. Die Nutzung nur der elektronischen Ausgaben ist für die deutschen Bibliotheken wegen der unterschiedlichen Besteuerung problematisch. Die Verlage verändern die Berechnungsbasis für die Nutzung inzwischen weg von den Abonnements hin zur Erhebung der potentiellen Nutzer oder zur Bezahlung des Einzelartikels. Aber diese Modelle führen nach aller Erfahrung zu weiteren Kostensteigerungen. Und die Konsortialabschlüsse führen zu einer Trennung der Kostenebene, die die Basiskosten der Bibliotheken für die Abonnements sowie zusätzliche Kosten für weitere Leistungen wie Zugang zur elektronischen Version, Cross-Access und Additional Access umfasst und zu zusätzlichen Nutzungsmöglichkeiten führt.

Wenn das in Zeitschriften vermittelte Wissen Ware ist und der Zeitschriftenmarkt ein Wettbewerbsmarkt wäre, müssten die Preise der Zeitschriften von der Qualität abhängen. Als Qualitätsmaße kommen die Impact-Faktoren, die Nutzungen oder die Zitate in Frage. Alle drei Faktoren haben keine oder nur eine schwache Korrelation zur Preisentwicklung. Und die Preise für alle Zeitschriften, unabhängig von diesen Leistungsfaktoren, sind in ähnlichem Umfang in den letzten Jahren im Preis gestiegen. Veränderungen der Impact-Faktoren zwischen minus zwanzig und plus siebzig Prozent führten zu Preissteigerungen im Beobachtungszeitraum von achtzehn Prozent. Daraus ist zu folgern, dass die Preise unabhängig von Marktmechanismen gesetzt werden. Der Preisbildungsprozess ist nicht darstellbar.

Was läuft falsch? - Das Subskriptionsmodell führt dazu, dass die Verleger ih Geld lange vor der Leistungserbringung bekommen. Das wäre auf einem Wettbewerbsmarkt nicht möglich. Wir haben neue Techniken, neue Nachfrage- und Vertriebsformen, aber alte Marktstrukturen.

Was läuft falsch! - Überhöhte Preise, Knebelverträge, Einschränkung der Archivrechte und scheinbar innovative Kosten- und Preismodelle treiben die Preise in die Höhe. (ACS hat angekündigt künftig auch das BSP des Abnehmerlandes in die Kalkulation einzubeziehen. Auch dies wird wohl kaum zur Preisdämpfung führen.) Und bei den Open-Choice-Angeboten zahlen die Autoren nochmals zusätzlich.

Die Folge ist, dass Open Access zu einer echten Alternative wird, weil hier der Reputationskreislauf und der Kostenkreislauf annähernd deckungsgleich werden Aber der Aufbau von Open-Access-Zeitschriften erfordert hohe Anfangsinvestitionen, da die Infrastruktur für die Begutachtungsverfahren erst aufgebaut werden muss, während es mit der Distribution und der Vermarktung keine Probleme gibt da diese wegfällt.

Es gibt folgende Zukunftsmöglichkeiten:

- es gibt kaum Veränderungen.

- Der Markt wird fragmentiert in Kaufzeitschriften, Open-Access-Zeitschriften und Pay-per-View-Vertrieb.

- Open-Acces ist emotional nicht durchsetzbar.

- Open-Access ist das Modell der Zukunft.

- Beim Subskriptionsmodell bezahlen die Bibliotheken die Verlage.

- Beim Open-Access-Modell bezahlen die Autoren die Verlage bzw. Gesellschaften, aber die Nutzung ist kostenfrei.

Ohne Verlage kann die Begutachtung über die "Learning Society" intern oder nachträglich und öffentlich erfolgen. Die fachlichen Institutionen müssten diese Strukturen aufbauen. (In einigen Disziplinen gibt es diese schon.) Neue Strukturen sind denkbar, müssen aber organisiert werden.

In der Diskussion wurde benannt,

- dass in einigen Bereichen wie z.B. Wikipedia und bei der Software lizenzfreie Produkte durchaus erfolgreich sind.

- und dass in der Informatik Zeitschriften eingehen, weil die Wissenschaftler zunehmend Server statt Zeitschriften zur Publikation nutzen. 
Als dritter Redner sprach Herr Dr. Wulf D. von Lucius vom Verlag Lucius und Lucius in Stuttgart unter der Überschrift: „Struktureller Wandel in der Wissenschaftskommunikation. Veränderte Rollen und Funktionen der Glieder der Informationskette".

Herr Lucius führte an, dass das Schreiben der Manuskripte schon immer von den Hochschulen bezahlt worden wäre ${ }^{2}$ und die Verlage alle Dienste nach de Manuskripterstellung bezahlen würden. Die Nutzung der eigenen Produkte durch die Wissenschaftler und ihre Institutionen sei unbestritten und für die Verlage auch kein Problem. Ein Bezahlen für das Veröffentlichen wie das Open-Access-Model es vorsehe führe zu einer Steuerung der Veröffentlichungen durch die öffentliche Hand. Diese Steuerung solle besser über den Markt durch die Verlage und das bei ihnen angesiedelte Begutachtungsverfahren erfolgen. Open Access in der Hand der Verlage könne funktionieren, aber die Wissenschaftler wären nicht in der Lage. dies selbst zu organisieren. Er kann sich vorstellen, dass die Wissenschaftler ihre Artikel auf die eigene Homepage oder auf die der Hochschule stellen, aber diese dürften nicht durch eine eigene Suchmöglichkeit erschlossen werden, da dies das Verwertungsrecht der Verlage schädigen würde. Die Basis der Verlagstätigkeit se auf absehbare Zeit die Herstellung der gedruckten Zeitschriften, da diese Grundlage die Wirtschaftlichkeit der Verlage sicherstelle.

Im vierten Beitrag steilte Professor Dr. Ernst-Ludwig von Thadden von der Universität Mannheim die Frage: „Funktioniert der Markt für wissenschaftliche Zeitschriften? Kann er funktionieren?".

Zeitschriftenartikel werden von Wissenschaftlern für Wissenschaftler geschrieben, und diese Beiträge zur wissenschaftlichen Diskussion müssen auf den Weg gebracht werden. Er selbst ist in diesem Bereich in drei Funktionen tätig, als Wissenschaftler, als Herausgeber einer wissenschaftlichen Zeitschrift und als Autor und Leser. Und als Wirtschaftswissenschaftler interessiert inn, wie der Markt für wissenschaftliche Zeitschriften funktioniert.

Märkte sind dadurch definiert dass es ein Gut und daran interessierte Konsumenten gibt. Das Gut ist der Transport wissenschaftlicher Inhalte, nicht diese selbst. Zeitschriften transportieren diese Inhalte, schaffen sie aber nicht. Die Konsumenten sind die Leser und die Autoren der Beiträge. Produzenten dieses Gutes "Transport" sind die

- Verlage mit Gewinnerzielungsabsicht (For Profit = FP)

- die Gesellschaften ohne Gewinnerzielungsabsicht (Not For Profit = NFP),

- sowie Verlage (FP), die Zeitschriften im Auftrag von Gesellschaften (NFP) herausgeben.

2 Dies ist meines Wissens falsch: Noch zwischen den Kriegen gab es Wissenschaftler die von den Honoraren für ihre Publikationen lebten.
Die Aufgabe der Zeitschriften ist die

- Verbreitung von Wissen (Transport) und

- die Zertifizierung von Wissen (durch Herausgeber und Begutachtung)

In den Wirtschaftswissenschaften gibt es wie in einigen anderen Wissenschaften auch eine brutale Hierarchie der Zeitschriften, die weltweit gilt. Da Wirtschaft überall gleich funktioniert, gibt es hier keine regionalen Differenzierungen mehr. Für den Wissenschaftler relevant ist einzig, welchen Rang die Zeitschrift hat, in der sein Beitrag veröffentlicht wird.

Die zentrale These des Beitrages ist, dass das Instrument Zeitschriften für die Verbreitung von Wissen immer unwichtiger, für die Zertifizierung des Wissens immer wichtiger wird.

Für die Verbreitung gibt es heute bessere Instrumente: In den Wirtschaftswissenschaften, die heute weltweit vernetzt sind, gibt es für den Wissenstransport beispielsweise Nachrichtendienste und spezielle Zeitschriften:

Nachrichtendienste sind das z.B. SSRN und das RePec. Das SSRN = Social Science Research Network ist ein Nachrichtendienst, in den die Wissenschaftler ihre Diskussionspapiere einstellen und das den Abonnenten täglich etwa 10 neue Beiträge liefert. In diesem SSRN erscheinen die Beiträge etwa drei bis fünf Jahre, bevo sie als fertige Papiere in einer Zeitschrift erscheinen. Das RePec ist eine Suchmaschine, die die Homepages der beteiligten Wissenschaftler absucht und alle dort neu eingestellten Beiträge anzeigt.

Spezielle Zeitschriften sind die

- BEPress (=Berkeley Electronic Press) oder

- Economics (Institut für Weltwirtschaft, Kiel).

BEPress hat das Begutachtungsverfahren so organisiert, dass die Artikel seh schnell erscheinen. Bei der Begutachtung werden sie in vier Qualitätsniveaus eingeteilt (sehr gut, gut, brauchbar und abgelehnt) und die angenommenen Artike werden in den entsprechenden Kategorien der BEPress nach sehr kurzer Begutachtungszeit eingestellt. Aber auch diese Zeitschriften haben für den Wissens transport vermutlich keine Zukunft, da dieser über die oben dargestellten Informationsdienste schneller erfolgt.

Ökonomische Anmerkungen zum Zeitschriftenmarkt: In den Wirtschaftswissenschaften ist wie in den meisten Wissenschaften das Urheberrecht kein Problem da die Autoren an der Verbreitung ihrer Ergebnisse und die Leser an der Information über diese Ergebnisse interessiert sind.

Forschungsergebnisse sind öffentliches Gut. Die/Verbreitung dieser Ergebnisse ist eine Dienstleistung, die heute mit geringen Kosten über das Internet erfolgen kann. Die gedruckte Zeitschrift ist dafür ein aussterbendes Medium. Die Zertifizie- 
rung dieser Ergebnisse andererseits erfordert viel Arbeitszeit und ist mit hohen Kosten verbunden. Diese Kosten werden über die Zeitschrift erwirtschaftet.

Herr von Thadden ist Mitherausgeber des "Journal of Financial Intermediation“, das von Elsevier verlegt wird. Dafür verwendet er zwei Arbeitstage je Woche. Er ist erstaunt darüber, dass das Land Baden-Württemberg inn dafür bezahlt, dass er diese Arbeit - für die Wissenschaftsgemeinschaft und für den Verlag - macht. In den USA wird die Herausgeberschaft von den Universitäten allerdings zusätzlich honoriert, indem dem Herausgeber von der Universität das Gehalt erhöht wird und er zusätzlich das Lehrdeputat reduziert bekommt.

Der Zeitschrift werden jährlich etwa 300 Artikel eingereicht. Für jeden Artikel werden zwei Gutachten angefertigt, und jeder Gutachter braucht für ein Gutachten jeweils zwei Tage. Damit erfordert diese Zeitschrift jährlich 600 Arbeitstage für die Begutachtung. Dieser Aufwand wird von den Arbeitgebern der Gutachter getragen. Was Elsevier den Gutachtern und Herausgebern für diese Arbeit vergütet, ist im Verhältnis zum Aufwand nicht der Rede wert. Die Zeitschrift kostet bei Elsevier Euro 500 je Abonnement. Die Abonnements der deutschen Bibliotheken reichen aus, die Drucklegung und den Vertrieb zu bezahlen. Die Erlöse aller außerhalb von Deutschland verkauften Abonnements sind für Elsevier Reingewinn.

Ein Zeitschriftentitel ist eine Marke. Das Renommee der Zeitschrift ist in vielen Jahren erarbeitet und kann nur zerstört werden, wenn die Herausgeber dies vorsätzlich wollen. Wenn sie dies fahrlässig geschehen lassen, greift der Verlag ein und wechselt die Herausgeber aus. Die Herausgeber der European Economic Review sind vor sechs Jahren bei Elsevier ausgestiegen, da sie mit der Politik von Elsevier nicht einverstanden waren und haben eine neue Zeitschrift gegründet, das Journal of European Economic Association. Diese wird im Auftrag der Association von MIT Press herausgegeben. Obwohl die besten Ökonomen Europas daran beteiligt sind und alles tun, das Renommee dieser neuen Zeitschrift aufzubauen, hat sie noch lange nicht den früheren Rang der European Economic Review erreicht. Dies dauert nach Einschätzung des Referenten 10 bis 20 Jahre.

Zeitschriften werden von den Bibliotheken nur gekauft, wenn sie von den Wissenschaftlern als Zertifizierungsinstrument anerkannt sind, d.h. wenn sie einen entsprechenden Rang haben. Das ökonomische Problem ist, dass der Zeitschriftenmarkt ein "Zweiseitiger Markt" ist, d.h. die Zeitschriften sind eine Plattform zwischen Autoren und Lesern, diese sind zum großen Teil identisch und es besteht eine gegenseitige Abhängigkeit der Erwartungen.

In der Diskussion wurde geklärt: Für die Verbreitung der Information sind Zeitschriften nicht mehr erforderlich, aber für die Zertifizierung des Wissens sind sie derzeit unabdingbar. Deshalb ist es nicht mehr erforderlich, dass die Bibliotheken aller an den jeweiligen Zeitschriften interessierten Wissenschaftlern diese physisch beschaffen, bearbeiten, lagern und wieder entsorgen. Aber es ist not- wendig, dass das Zertifizierungsverfahren sichergestellt wird. Wenn dies über die herkömmlichen Zeitschriften nicht mehr sichergestellt werden kann, muss die wissenschaftliche Gemeinschaft das Zertifizierungsverfahren auf andere Weise sicherstellen. Es würde ausreichen, wenn wenige Bibliotheken die Archivierung sicherstellen.

Zum Abschluss der Veranstaltung fand eine Podiumsdiskussion statt, die vom Prorektor für Forschung der Universität Mannheim Herrn Professor Dr. Peter Drewek geleitet wurde. An dieser nahmen die Referenten des Tages außer Herrn Dreier sowie die Informationswissenschaftlerin Frau Professor Angela Keppler und der Germanist Herr Professor Dr. Jochen Hörisch von der Universität Mannheim teil. In dieser Diskussion wurden folgende Sachverhalte geklärt oder benannt:

- Nicht nur das Ob, sondern auch das Wie des Wissenstransports ist dafür relevant, wie Wissen aufgenommen und verarbeitet wird. Sowohl beim Lesen gedruckter Informationen - besonders in der Bibliothek - wie auch beim Lesen am Bildschirm werden zusätzliche Informationen aufgenommen. Dies sind aber sehr unterschiedliche Informationen, die ihrerseits die Rezeption beeinflussen. Ebenso ist die Rezeption von Zeitschriftenbeiträgen und Büchern eine sehr unterschiedliche. Und in weiten Bereichen der Wissenschaft ist die Wissensweitergabe durch Bücher durchaus weiterhin relevant.

- Verlage haben bei den Zeitschriften wie bei den Büchern die Aufgabe der Selektion.

- Wenn Wissen eine Ware wäre, müsste es Knappheitspreise geben. Aber es gibt eine sehr große Produktionsmenge und trotzdem ist die "Ware Zeitschrift" immens teuer. Wissen ist heute ein Massenprodukt und bewertet werden eher Indexwerte als das Wissen bzw. die wissenschaftlichen Ergebnisse selbst. Der Glaube an die Messbarkeit durch die Zertifizierung behindert eher das echte Wissen und für spezifische Einzelinformationen gibt es keine Konkurrenz.

- Oder ist die Information das Massenprodukt und das Wissen ist etwas anderes? In der Informationsproduktion gibt es eine immense Betriebsamkeit, aber die Universität ist eigentlich dazu da, aufmerksam zu bleiben und Wissen zu vermitteln statt nur Informationsmengen. Hier sollte klares Denken und nicht nur Informationsrezeption vermittelt werden.

- Die Frage ist, wie die Produktion der Informationsmengen reduziert werden kann. Die Publikationsmengen sind durch die Wissenschaftler verursacht und müssten von diesen gesteuert werden. Solange der Erfolg von der Länge der Publikationslisten und der Rangordnung der Zeitschriften, in denen diese Publikationen erschienen, und nicht von den Inhalten der Publikationen abhängen, wird das Problem weiter anwachseh. Die Begutachtungsverfahren reduzieren die Publikationsmengen nur unzureichend, da letztendlich jedes Papier seine Zeitschrift findet. Und die Wissenschaftler veranlassen laufend die Gründung neuer Zeitschriften. 
- Die Begutachtungsverfahren und die Anforderungen für Veröffentlichungen in bestimmten Zeitschriften standardisieren einerseits die Veröffentlichungen. Andererseits werden in den Spitzenzeitschriften nur innovative Beiträge angenommen (?)

- Die Publikationsmengen und die technischen Möglichkeiten führen einerseits zu einer Informationsbreite in der Bevölkerung und Demokratisierung des Wissens, die in früheren Jahrzehnten nicht denkbar waren. Andererseits werden die Verlage bei den Zeitschriften wie bei den Büchern die Aufgabe der Selektion haben. Auch werden für die Rezeption Sekundärformen des Wissens entwickelt, die zur Selektion und Aufbereitung der Informationsmengen erforderlich geworden sind. Diese Selektionsmechanismen wie die Begutachtungsverfahren führen ihrerseits wieder zu einer Aristokratisierung des Wissens, wenn die Entscheidungsträger sich nach deren Ergebnissen richten. Wie fragwürdig diese Mechanismen sind, ist zum Beispiel an CHE-Ranking abzulesen, wenn in den Ergebnissen selbst auf die Fragwürdigkeit der Datenbasis hingewiesen wird.

- Die schnelle Verbreitung von Informationen über das Internet erfordert neue und schnellere Verfahren zur Wissensselektion als die herkömmlichen Begutachtungsverfahren. Auch müssen die Nutzer lernen, wie sie die dort gebotenen Inhalte selbst qualitativ bewerten können. Ansätze dazu kennt jeder, wenn man Informationen danach sortiert, wer sie erstellt und bereitstellt. Dies gilt für Wikipedia wie für jede Zeitung. Wikipedia hat den Vorteil, dass man den Meinungsbildungsprozess nachvollziehen kann, im Gegensatz zum Selektionsprozess bei den meisten Zeitschriften.

- Das Urheberrecht und die daraus abgeleiteten Verwertungsrechte sind die Basis der derzeitigen Bewertungsverfahren für Informationen. Es ist möglich, dass durch den Eintritt der Länder, die diese in der westlichen Wissenschaftswelt entwickelten Mechanismen nicht akzeptieren oder bewusst unterlaufen, diese Grundlagen auf Dauer nicht mehr tragfähig sind.

Als Ergebnis lässt sich feststellen, dass die heute gegebenen technischen Mög lichkeiten der weltweiten Beteiligung breiter Bevölkerungsschichten am Prozess der Informationserstellung und Informationsnutzung zu einer Demokratisierung des Wissens geführt und weiter führen wird. Dies hat zur Folge, dass die bisherigen Mechanismen der Bewertung und Bereitstellung von Wissen in Frage gestellt sind und in den nächsten Jahren vermutlich gravierende Veränderungen erfahren werden. 